\title{
Infestation of Narcissus poeticus by a scathophagid fly, Norellia melaleuca (Diptera: Scathophagidae)
}

\author{
GIUSEPPE CHICHIRICCÒ
}

Dipartimento di Scienze Ambientali, University of L’Aquila, Via Vetoio, I-67100 L’Aquila, Italy; e-mail: chichiri@univaq.it

Key words. Scathophagidae, Narcissus poeticus, Norellia melaleuca, phytophagy, reproductive cycles

\begin{abstract}
A little-known scathophagid fly is reported infesting the flowers and scapes of Narcissus poeticus growing in the Italian Central Apennines (AQ). This insect bores through the flower bud and lays an egg on the tepals. Both pistil and scape parenchymatous tissues provide the larva with nutrition, and the hollow stem a safe site for its metamorphosis. Infestation by this insect prevents the ovules from being fertilized as the larvae feed on the tisues in the style and so prevent the growth of pollen tubes.
\end{abstract}

\section{INTRODUCTION}

Scathophagidae (see Kato et al., 2006; Ovchinnikov, 2009) is a large family of flies well represented in the Palaearctic and Nearctic regions, with 250 species in 50 genera. Adults are predators on other Diptera or small invertebrates, in particular blow-flies. Larvae, depending on the species, are saprophagous, facultative or obligatory predators, or phytophagous on angiosperms of which they mine leaves, bore stems, enter corms, or feed on seeds in capsules. The larvae of $N$. spinipes mine the leaves and stem bases of Narcissus (Ovchinnikov, 2009), a geophyte of the family Amaryllidaceae largely used in horticulture, medicine, pharmacology and production of cosmetics (Hanks, 2002). Larvae of Diptera found within flowers of Narcissus poeticus L. growing in the Italian Central Apennines stimulated the present study on the life history of this insect and its effect on this plants reproduction.

\section{MATERIAL AND METHODS}

\section{Monitoring of infested plants and capture of insects}

Four plots, with 80 plants per plot, were established in the Rocche highlands (AQ), which extend over 2500 ha at an altitude of $1300 \mathrm{~m}$ a.s.l. in the Italian Central Apennines. At these plots, during April-May 2008, a total of 320 flowering plants were examined for signs of infestation by phytophagous insects. The pistil and stem of each plant were examined for the presence of larvae and a number of stems were enclosed in plastic transparent cylinders the tops of which were covered with nylon mesh to entrap the adult insects.

\section{Light and fluorescence microscopy}

For anatomical observations, pistils were fixed in acetic acid and alcohol (1:3), dehydrated in an ethanol series and embedded in Technovit 7100 resin (Kulzer). Sections were cut at 3-6 $\mu \mathrm{m}$ using a Reichert-Jung 2040 microtome, stained with $0.05 \%$ toluidine blue and observed under a Zeiss Axioplan 2 microscope equipped with a Leica DFC 350 FX digital camera.

To observe the pollen tubes within the ovary, pistils were stained with aniline blue, squashed and then the fluorescing pollen tubes (Kho \& Baer, 1968) were observed under a fluorescence microscope.

\section{Scanning electron microscopy}

Ovaries were dissected, treated with $\mathrm{CO}_{2}$, fixed in acetic acid and alcohol (1:3), dehydrated in an ethanol series and critical point dried in $\mathrm{CO}_{2}$. They were then gold-coated using Balzer's SCD 040 sputtering, and viewed using a Philips 505 scanning electron microscope

\section{RESULTS}

\section{Invasion of plant tissues by $\boldsymbol{N}$. melaleuca}

The infested flower buds of $N$. poeticus are marked by small holes in the enveloping bracts and tepals, and inside each bud is usually one developing egg on the innermost tepals (Fig. 1a). On hatching the larva (Fig. 1b) locates the pistil and penetrates it (Fig. 2a) just below the papillate stigma. The style (Figs 2a-d) is made up of parenchymatous tissue along which three symmetrical vascular bundles extend peripherally and a central canal lined with transmitting tissue, which provides a nourishing medium for the pollen tubes. The larva mines a tunnel parallel to the stylar canal by feeding on the parenchymatous tissue between two vascular bundles without damaging them (Fig. 2b). In the lower half of the style the larva continues its tunnel in the stylar canal (Fig. 2c). A single tunnel and a single larva were found in each style (Fig. 2b) except in the few cases when the first larva died (Fig. 2d).

The larva tunnels into the ovary longitudinally (Figs 1c, d) through the nectariferous ovarian septa or the axile column without damaging the vascular bundles or the ovules enclosed by the septa in the three ovarian locules, each with on average 32 ovules.

The ovary is potentially the site for further ovipositions and the resultant larvae may tunnel the ovarian septa transversally and eat some of the ovules. However if the pistil already hosts a larva, the egg does not develop (Fig. 1e).

After crossing the ovary, the larva tunnels into the pithy floral scape (Fig. 1f) and quickly down the hollow stem. At the bottom of the hollow stem the larva excavates an annular canal in the inner stem wall (Fig. 1g), and with its head facing upwards pupates within the last larval exuvium. Exuvia split along the rim of the flattened apical side, except in the highest tract, which functions as a hinge (Fig. 1h). When the plant wilts (June-July), the stem detaches where the larva made the annular tunnel, which allows the mature insect to leave the host plant. Both the male and female flies that emerged from the exuvia in hollow stems (Fig. 1i) were identified as Norellia melaleuca Loew (Scathophagidae). 

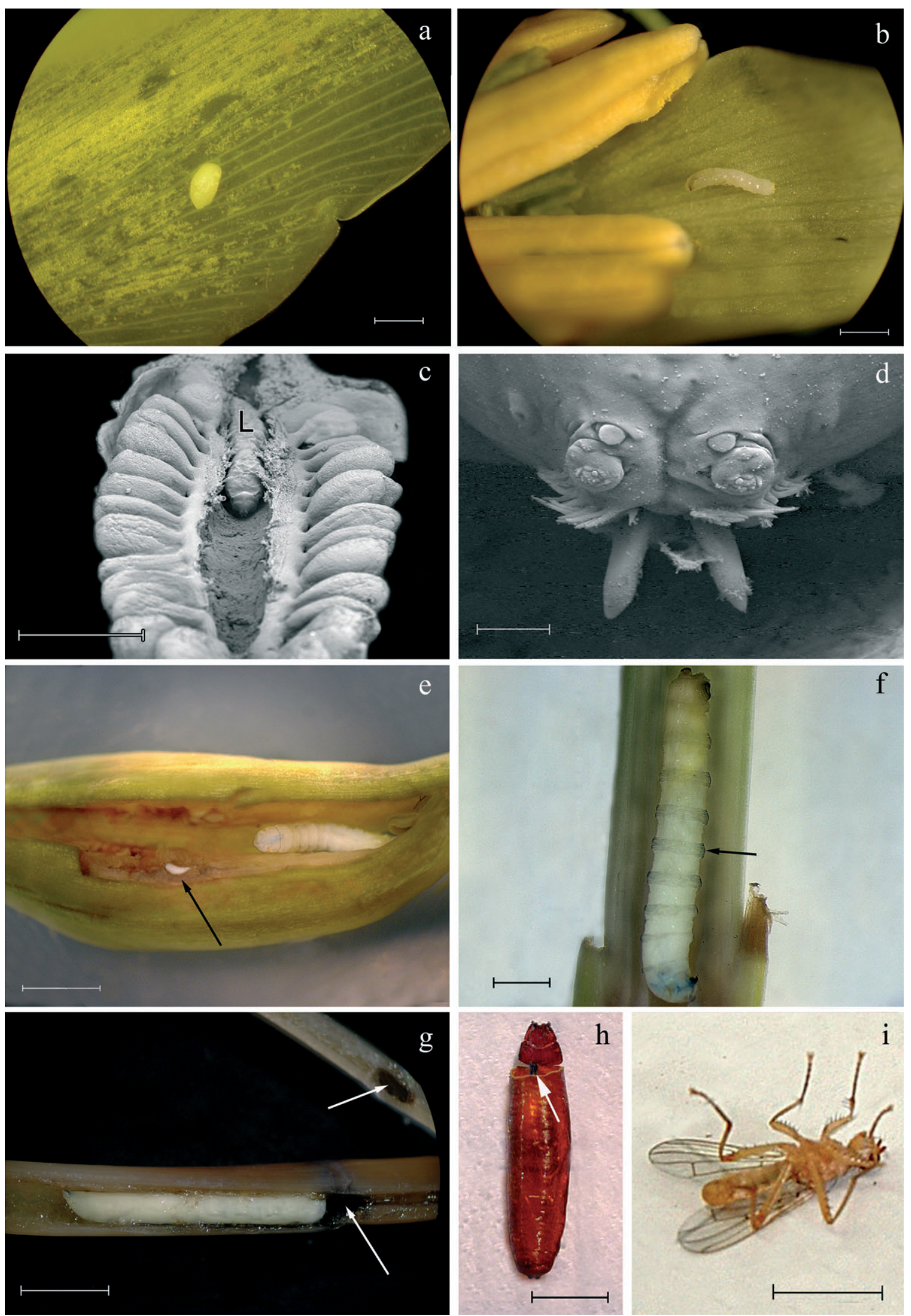

Fig. 1. Developmental stages, from egg to adult, of Norellia melaleuca recorded using a stereomicroscope (a, b, e-i) and scanning electron microscope (c, d). Developing egg (a) and larva (b) on the tepal of a flower bud; larva within the tunnelled ovarian septum (c) and magnified picture of its head (d); e - ovary containing a well-developed larva and an undeveloped egg (arrow); f - larva at the base of the scape, the arrow indicates the rings of bristles; $g$ - larva at the bottom of a hollow stem, the arrows indicate the subcavity; h, exuvium from which an adult has emerged, the arrow indicates the mandibles of the larva; i, adult insect some days after emerging from an exuvium. $\operatorname{Bar}=0.7 \mathrm{~mm}(\mathrm{a}, \mathrm{b}) ; 1 \mathrm{~mm}(\mathrm{c}) ; 20 \mu \mathrm{m}(\mathrm{d}) ; 1.5 \mathrm{~mm}$ (e); $1.0 \mathrm{~mm}$ (f); $4.5 \mathrm{~mm}(\mathrm{~g}) ; 2.5 \mathrm{~mm}(\mathrm{~h}) ; 4.5 \mathrm{~mm}$ (i). 

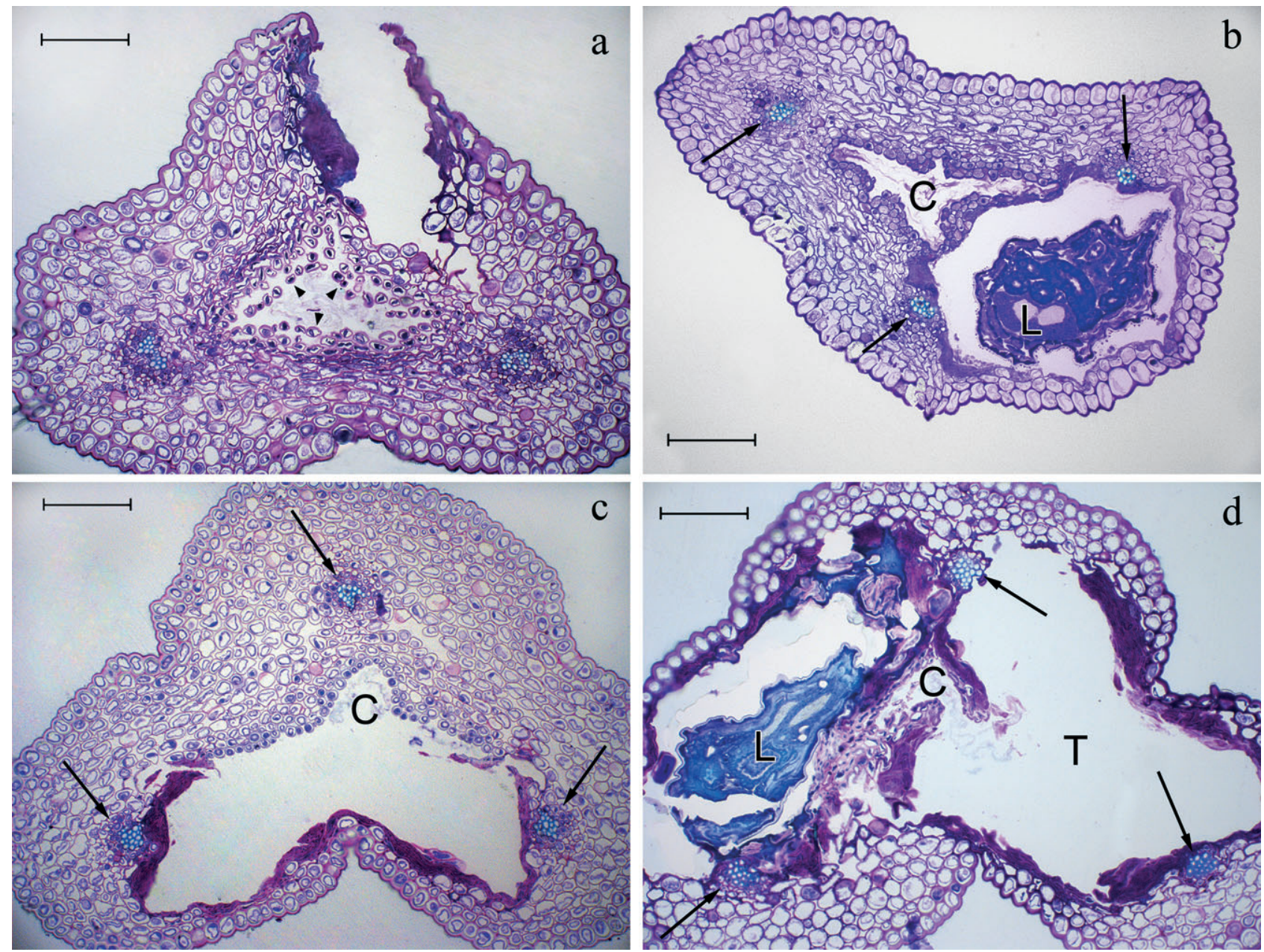

Fig. 2. Cross sections through tunnelled styles. a - horizontal tunnel at the point of entry of the larva, note the pollen tubes (arrowheads) and the mucilaginous nourishing medium in the stylar canal; b - tunnel with the larva, adjacent to the stylar canal; $\mathrm{c}$ - tunnel has entered the stylar canal, pollen tubes are absent; $d$ - two tunnels, one containing the remains of a dead larva and the other a second larva. $\mathrm{C}$ - stylar canal; $\mathrm{L}$ - larva; $\mathrm{T}$ - tunnel. The arrows indicate the vascular bundles. Bars $=100 \mu \mathrm{m}$.

\section{Plant vulnerability and growth of pollen tubes}

Most plants in the field (80.8\%) are infested with fly larvae; 258 plants out of 320 plants examined. The larvae did not interfere with either flowering, or pollination as germinated pollen was observed in pistils attacked by larvae (Fig. 2a). In the lower part of a style where the larva tunnels into the stylar canal, however, pollen tubes were not observed (Fig. 2c). In this case, the fluorescence microscopy revealed that no pollen tubes reached the ovules.

In contrast, fluorescence microscopy revealed that there were numerous pollent tubes in the pistils and ovules of plants growing in the field that were not attacked (not reported), with from 15 to 59 pollen tubes in each ovary.

\section{DISCUSSION}

Narcissus plants are attacked by Syrphid (Hodson, 1932; Kato et al., 2006) and Scathophagid (Ovchinnikov, 2009) flies, the larvae of which feed on the bulbs and leaves, respectively. Here is reported for the first time a Scathophagid fly the larvae of which feed on the pistils and scapes of Narcissus.

The female insect has an ovipositor that is well adapted to insert eggs into the buds of its host plant. The stigma of Narcissus, however, is provided with papillae, which may hinder larval movement. This may be the reason why the larvae bypass the stigma and enter by tunnelling into the style. Within the style, the larva feeds on the soft parenchymatous tissue and avoids damaging the vascular bundles. By tunnelling in this way it is possible that larvae do not cause the pistil to wilt. The style is mined by a single larva but if it dies, another larva may develop, suggesting that there are cues indicating the presence of a competitor (see De Moraes et al., 2001).

Within the ovary, the tunnelling causes little damage when the tunnels run longitudinally through the nectariferous ovarian septa. In addition to being a route for the larva it can (see Harvey et al., 2000) also prevent the development of more than one larva in a pistil.

The hollow stem is a good site for metamorphosis and the annular mining by the larva at the base of the stem facilitates subsequent adult emergence.

The insect infests plants by tunnelling down pistils before they are pollinated. The receptivity of the style to pollen is preserved but the pollen tubes are unable to reach the ovules because where the stylar canal is tunnelled the nourishing medium needed for the pollen tubes to reach the ovules is eaten by the larva. As a result, the fertilization of ovules is prevented. However, plant growth and vegetative propagation are not affected, and the bulbs produce bulb clusters just like the non infested plants.

In conclusion, $N$. melaleuca appears to be well adapted to feeding on the reproductive and vegetative structures of $N$. 
poeticus and is widely distributed throughout the Italian Central Apennines. In feeding on the reproductive and vegetative structures this insect prevents the sexual reproduction of its host plant without great damages to these structures. This limits both the inducible response of the plant and its ability to produce resistant genotypes, on the one hand, the decrease in plant fitness may favour genotypes that are better defended.

ACKNOWLEDGEMENTS. The author is grateful to I. Rivosecchi for the identification of the insect and Parco SirenteVelino, Rocca di Mezzo (AQ), for funding the project.

\section{REFERENCES}

De Moraes C.M., Mescher M.C. \& Tumlison J.H. 2001: Caterpillar-induced nocturnal plant volatiles repel conspecific females. Nature 410: 577-580.
Hanks G.R. 2002: Narcissus and Daffoild: The Genus Narcissus. Taylor \& Francis, London, New York, 452 pp.

Harvey J.A., Corley L.S. \& Strand M.R. 2000: Competition induces adaptive shifts in caste ratios of a polyembryonic wasp. Nature 406: 183-186.

Hodson W.E.H. 1932: The large Narcissus fly, Merodon equestris, Fab. (Syrphidae). Bull. Entomol. Res. 23: 429-448.

Kato M., Kaoru T. \& Kawakita A. 2006: Pollinator and stemand corm-boring insects associated with mycoheterotrophic orchid Gastrodia elata. Ann. Entomol. Soc. Am. 99: 851-858.

KHO Y.O. \& BAER J. 1968: Observing pollen tubes by means of fluorescence. Euphytica 17: 298-303.

OvchinNIKov A.N. 2009: The ovipositor morphology in the members of the family Scathophagidae (Diptera) with reference to their biology. Entomol. Rev. 89: 623-636.

Received February 8, 2010; revised and accepted March 26, 2010 